\title{
Trans sodium crocetinate with temozolomide and radiation therapy for glioblastoma multiforme
}

\author{
John L. Gainer, PhD, ${ }^{1,2}$ Jason P. Sheehan, MD, PhD, ${ }^{3}$ James M. Larner, MD, ${ }^{4}$ and \\ David R. Jones, MD ${ }^{1,5}$ \\ 'Diffusion Pharmaceuticals LLC; Departments of ${ }^{3}$ Neurological Surgery and ${ }^{4}$ Radiation Oncology, ${ }^{2}$ University of Virginia, \\ Charlottesville, Virginia; and ${ }^{5}$ Division of Thoracic Surgery, Memorial Sloan Kettering Cancer Center, New York, New York
}

\begin{abstract}
OBJECTIVE A new drug, trans sodium crocetinate (TSC), has been developed to enhance the delivery of oxygen to hypoxic tissues. Cancerous tumors, such as glioblastoma multiforme (GBM), are very hypoxic, and it has been suggested that radiation therapy $(\mathrm{RT})$ is more beneficial if tumors are better oxygenated. A Phase $\mathrm{I} / \mathrm{II}$ clinical trial was conducted to determine the effect of adding TSC to RT sessions.
\end{abstract}

METHODS An open, single-arm clinical trial incorporating the standard of care (SOC) for GBM was conducted at 18 clinical sites. There were 6 weeks of RT consisting of 2 Gy/day for 5 days/week, beginning after an initial resection or stereotactic biopsy to confirm GBM. Temozolomide (TMZ), $75 \mathrm{mg} / \mathrm{m}^{2}$, was given before each RT session. The TSC, $0.25 \mathrm{mg} / \mathrm{kg}$, was intravenously administered around 45 minutes before an RT session 3 days/week, usually on Monday, Wednesday, and Friday.

A Phase I run-in period included 2 cohorts. The first cohort contained 3 patients who were given a half dose of the intravenous TSC (that is, $0.25 \mathrm{mg} / \mathrm{kg}$, 3 times per week for only the first 3 weeks of RT). After a Safety Monitoring Committee (SMC) had verified that no dose-limiting toxicity (DLT) had occurred, a second cohort of 6 patients was given the same dosage of TSC but for the full 6 weeks of RT. After the SMC verified that no DLTs had occurred, Phase II began, with the administration of the full 18 doses of TSC. Fifty additional patients were enrolled during Phase II.

Following the completion of RT, the patients rested for a month. After that, SOC TMZ chemotherapy (150-200 mg/m²) was administered for 5 days of the 1st week of 6 monthly cycles. No TSC was administered during this chemotherapy phase or later in the trial. Any other follow-up therapies were administered at the discretion of the individual investigators. RESULTS Kaplan-Meier analysis showed that $36 \%$ of the full-dose TSC patients were alive at 2 years, compared with historical survival values ranging from $27 \%$ to $30 \%$ for the SOC. Survival for the biopsy-only subset of patients was $40 \%$, as compared with $42.9 \%$ for those patients having a complete resection before treatment. In addition, 2 of the 3 Phase I, Cohort 1 patients survived at 2 years. Contrast MRI data suggested that considerable pseudoprogression had occurred.

Both Karnofsky Performance Status (KPS) scores and quality of life (QOL) questionnaires indicated that a good quality of life existed for most patients throughout the trial. No serious adverse events occurring in the trial were attributed to TSC.

CONCLUSIONS This trial contained a single arm consisting of 59 patients. The results strongly suggested that adding TSC during RT is beneficial for the treatment of GBM. Trans sodium crocetinate offers a novel, easily implemented way to combat hypoxia in tumor tissue.

Clinical trial registration no.: NCT01465347 (clinicaltrials.gov)

http://thejns.org/doi/abs/10.3171/2016.3.JNS152693

KEY WORDS glioblastoma; trans sodium crocetinate; clinical trial; hypoxia; radiation therapy; oncology

ABBREVIATIONS DLT = dose-limiting toxicity; EORTC = European Organisation for Research and Treatment of Cancer; GBM = glioblastoma multiforme; KPS $=$ Karnofsky Performance Status; PFS = progression-free survival; QLQ-BN20 = 20-Item Quality of Life Questionnaire-Brain Neoplasm; QLQ-C30 = 30-Item Core Quality of Life Questionnaire; RT = radiation therapy; SMC = Safety Monitoring Committee; SOC = standard of care; TMZ = temozolomide; TSC = trans sodium crocetinate.

SUBMITTED November 22, 2015. ACCEPTED March 4, 2016.

INCLUDE WHEN CITING Published online May 13, 2016; DOI: 10.3171/2016.3.JNS152693. 
$\mathrm{G}$ LIOBLASTOMA multiforme (GBM), the most common primary brain tumor in adults, is said to be highly invasive and virtually incurable. Even with the standard of care (SOC) of maximum safe resection followed by temozolomide (TMZ) and radiation therapy (RT), only around $26 \%$ of patients are alive at 24 months. ${ }^{14}$

Like other solid tumors, GBMs exhibit very low oxygen levels, and hypoxic tumors are known to be more resistant to RT. ${ }^{12}$ Therefore, the hypothesis that increasing tumor oxygen levels could improve the efficacy of RT has frequently been suggested.

Trans sodium crocetinate (TSC) was developed at the University of Virginia, under the sponsorship of the United States Office of Naval Research, as a treatment for battlefield casualties. Since the majority of battlefield casualties occur due to hemorrhagic shock resulting in severe hypoxia, TSC was designed to be an easily implemented systemic treatment. In animals, TSC has been shown to increase whole-body oxygen levels during hypoxia caused by extreme blood loss 9 as well as in other hypoxic conditions. ${ }^{3}$ The mechanism of action for TSC is further described later in this report.

Thus, it seemed reasonable to combine the antihypoxia drug TSC with the RT portion of the SOC for GBM. Studies with a rat model of GBM have also suggested that TSC might offer a novel beneficial treatment; ${ }^{11}$ thus, a Phase I/ II clinical trial was conducted.

\section{Methods}

Diffusion Pharmaceuticals LLC, Charlottesville, Virginia, conducted an open-label, multicenter, single-arm Phase II study, including a Phase I lead-in, combining TSC with RT and TMZ for the treatment of newly diagnosed GBM. The trial, referred to as the Safety and Efficacy Study of Trans Sodium Crocetinate (TSC) With Concomitant Radiation Therapy and Temozolomide in Newly Diagnosed Glioblastoma (GBM), was registered with the ClinicalTrials.gov database (http://www.clinicaltrials.gov), and its registration no. is NCT01465347. The trial was coordinated by INC Research, Raleigh, North Carolina. All data reported here were collected and analyzed by INC Research, independent of Diffusion Pharmaceuticals.

Fifty-nine patients with newly diagnosed GBM were enrolled between January 2012 and March 2013. The trial began at the University of Virginia Medical Center and was later expanded to 17 other sites (see list in Appendix). Starting within 5 weeks after tumor resection or needle biopsy only, patients received SOC RT (2 Gy/day for 5 days/week for 6 weeks) and TMZ (75 mg/m² for 5 days/ week for 6 weeks). In addition to the SOC, TSC $(0.25 \mathrm{mg}$ / $\mathrm{kg}$ ) was intravenously administered 3 times a week, usually on Monday, Wednesday, and Friday, about 45 minutes prior to the RT sessions.

The trial began with a Phase I run-in period to establish the safety of dosing TSC concurrently with RT and TMZ. Three patients received TSC for only the first 3 weeks of the RT sessions. After a Safety Monitoring Committee (SMC) had determined that the TSC caused no dose-limiting toxicity (DLT) 10 weeks after this combined therapy, 6 additional patients received TSC for the entire 6 weeks of RT at the same dosage per week. Once the SMC again judged that no DLTs occurred within a 10-week period after the drug had been administered, Phase II was begun, enrolling 50 additional patients, all receiving the established safe dosing regimen of $0.25 \mathrm{mg} / \mathrm{kg} \mathrm{TSC}$, intravenously, 3 times a week, about 45 minutes prior to RT. Informed consent was received from all patients treated, and each of the institutional review boards of the clinical sites approved the protocol.

Four weeks after the completion of RT, patients began chemotherapy with TMZ (150-200 mg/m²) for 5 days of the 1st week of a 4-week cycle, continuing for 6 such cycles. No TSC was administered during this period of chemotherapy. After chemotherapy, the patients were treated as per the discretion of the clinical site.

During each of the subsequent patient visits, which usually occurred every 8 weeks, data consisting of contrast MRI, Karnofsky Performance Status (KPS) scores, and answers to 2 quality of life questionnaires, the European Organisation for Research and Treatment of Cancer (EORTC) 30-Item Core Quality of Life Questionnaire (QLQ-C30) and the EORTC 20-Item QOL-Brain Neoplasm (QLQ-BN20), were collected. Comparative tumor areas were calculated from the maximum diameters and lengths shown on the MR images. Patients were followed up for 110 weeks after their treatment began.

\section{Results}

In Table 1, the 59 patients entered in the current trial are compared with those in the Stupp et al. study, ${ }^{14}$ which established the SOC treatment for GBM. The safety aspects of TSC in all 59 patients are shown in Table 2. Overall survival was analyzed using Kaplan-Meier statistics at 1 and 2 years; the results are compared with those from the Stupp trial in Table $3 .^{14}$

In the current trial, about the same number of patients underwent biopsy only (15) and complete resection (14). The comparison of survival at 2 years between those 2 groups is depicted in Fig. 1.

Fifty-six patients received the full-dose TSC therapy. Of those patients, 4 did not live long enough to have an MRI study after baseline, 1 patient was censored, and 14

TABLE 1. Demographic characteristics of patients at baseline

\begin{tabular}{llc}
\hline \multirow{2}{*}{ Characteristic } & \multicolumn{2}{c}{ No. (\%) } \\
\cline { 2 - 3 } & TSC Trial & Stupp Trial \\
\hline Total no. of patients & 59 & 287 \\
\hline Age in yrs & & 56 \\
Median & 59 & $19-70$ \\
Range & $31-84$ & \\
Sex & & $185(64)$ \\
Male & $33(56)$ & $102(36)$ \\
Female & $26(44)$ & \\
Extent of surgery & & $48(17)$ \\
Biopsy only & $15(27)$ & $239(83)$ \\
Debulking & $41(73)$ & $113(47)$ \\
Complete resection & $14(34)$ & $126(53)$ \\
$\quad$ Partial resection & $27(66)$ &
\end{tabular}


TABLE 2. Safety summary

\begin{tabular}{cc}
\hline \multicolumn{1}{c}{ Parameter } & No. \\
\hline Serious adverse event & \\
\hline Patients w/ serious adverse events & 12 \\
\hline Associated w/ TSC & 0 \\
\hline Adverse event & 52 \\
\hline Patients w/ adverse events & 10 \\
\hline Associated w/ TSC & \\
\hline Occurring in >1 patient & 4 \\
\hline Fatigue & 2 \\
Confusion & 2 \\
\hline Injection site &
\end{tabular}

patients underwent complete resections. Thus, 37 patients had either partial resection or no resection (biopsy only) and their tumors could be followed over time. Estimates of relative lesion sizes in these 37 tumor-bearing patients were obtained on contrast MRI by multiplying the maximum tumor diameter by the maximum tumor length. All MR images were first read at the clinical site and then reread by a central facility (Biomedical Systems). The latter set of data was used for the analysis below, with changes in the mean tumor size indicated in Fig. 2.

The reduction in mean tumor size over the duration of the trial could be the result of patients dying, since the number of reported MRI studies decreased as the trial progressed. Thus, we calculated the percentage of tumor size reduction in all 37 tumor-bearing patients (Fig. 3). The tumors in 27 (73\%) of these 37 tumor-bearing patients regressed during this trial. The addition of TSC to the SOC resulted in tumor reductions greater than $40 \%$ in 21 of the 37 patients. Perhaps the most significant result is the complete disappearance (100\% reduction) of tumor in 11 patients (30\%).

Median KPS scores for the duration of the trial are shown in Fig. 4. The numbers in parentheses represent the number of patients whose data were reported by the clinical sites and do not necessarily correspond to the number of patients alive at the time.

It should be noted that TSC was given only for the 6 weeks during which RT was also delivered. During that period, median KPS scores improved as shown in Fig. 5.

Quality of life as assessed by patient questionnaires was difficult to measure over the entire duration of this trial because responses to the questionnaires were not always reported, especially for the latter weeks of the trial. Figure 6 shows the scores over a large portion of the trial during which sufficient data were available to provide meaningful results. These data are subsets of various questions on

TABLE 3. Overall survival from Kaplan-Meier analysis

\begin{tabular}{cccccc}
\hline & \multicolumn{2}{c}{ TSC Trial } & & \multicolumn{2}{c}{ Stupp Trial } \\
\cline { 2 - 3 } \cline { 5 - 6 } Time & OS & $95 \% \mathrm{Cl}$ & & OS & $95 \% \mathrm{Cl}$ \\
\hline $1 \mathrm{yr}$ & $71.2 \%$ & $59.2,83.1$ & & $61.1 \%$ & $55.4,66.7$ \\
\hline $2 \mathrm{yrs}$ & $36.3 \%$ & $23.6,49.1$ & & $26.5 \%$ & $21.2,31.7$ \\
\hline \multicolumn{2}{c}{ OS = overall survival. } & & & &
\end{tabular}

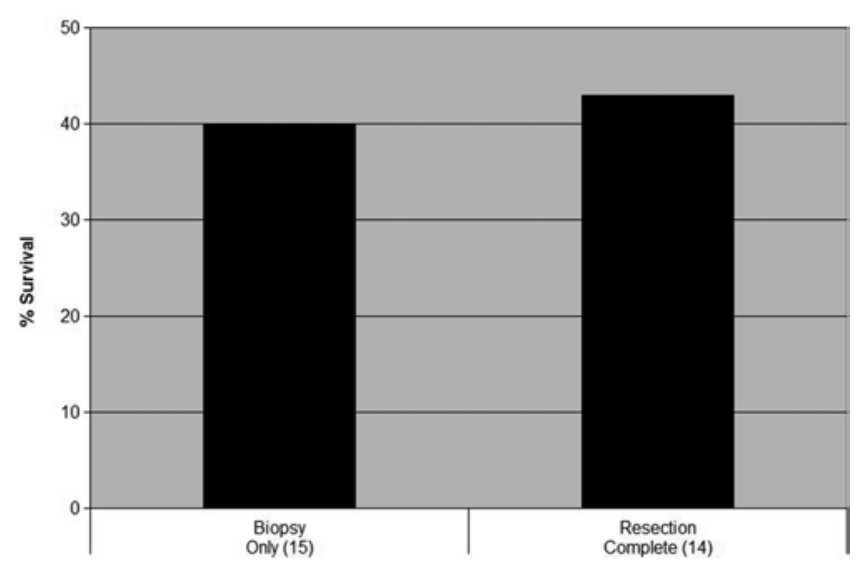

FIG. 1. Survival at 2 years in biopsy-only and complete-resection patients.

the EORTC QLQ-C30. Higher scores on the functional and global health subsets are good, while a lower score on the symptomatic subset is good.

\section{Discussion \\ Safety}

In comparing some demographic data from the current trial with those from the trial that established the SOC for newly diagnosed GBM,${ }^{14}$ we found that the median age in both trials was similar (Table 1). However, the current trial actually enrolled 5 patients older than 70 years $(8.5 \%$ of total patients), while the historic trial enrolled none in that age group. Overall survival with GBM has been inversely correlated with age, suggesting that the current trial may have been expected to produce less beneficial results. In addition, although it is widely recognized that hematological complications can occur with TMZ administration, such adverse effects have been reported to be worse in older patients..$^{10}$ In the current trial, no serious adverse events were associated with TSC in any patients (Table 2), and neither did any complications arise due to TMZ. Thus, adding TSC to the SOC would appear to increase the ability of patients to tolerate the TMZ treatment, even the older ones.

A previous Phase II clinical trial of TSC for a very different indication, peripheral artery disease, also showed no serious adverse effects attributable to TSC in 48 patients. ${ }^{8}$ From all available clinical data, it would seem that TSC is a safe drug. It is totally novel, and perhaps its safety profile is related to a mechanism of action unlike that of any other pharmaceutical agent. That mechanism of action is briefly discussed below.

Obviously, safety and tolerability are the major considerations when proposing a new drug treatment. Since TSC has been shown to be very safe in 3 clinical trials to date and well tolerated by patients, some indications of efficacy are also described in the sections below. There was no control arm in this trial, but the current SOC was established by a historical trial. ${ }^{14}$ Thus, that trial was used as a comparison here, but a Phase III trial containing an $\mathrm{SOC}$ arm will begin in the upcoming year. 


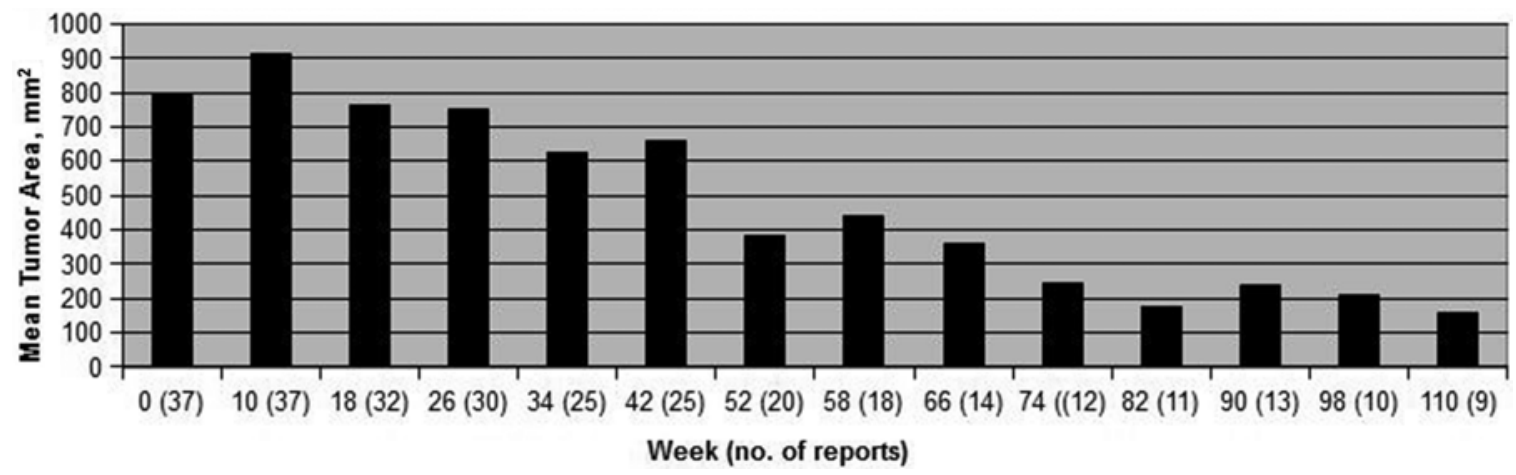

FIG. 2. Mean tumor area changes in 37 tumor-bearing patients.

\section{Overall Survival}

Overall survival at 1 and/or 2 years is the main end point for most GBM trials. However, comparative KaplanMeier curves were not possible in the present study since the individual data for the historical trial were not available. Using the values reported for certain time points in the SOC analysis, as shown in Table 3, we determined that survival was $10 \%$ greater in the TSC trial at both 1 and 2 years than the rate in the historical trial, which had established the SOC for GBM in 2005. ${ }^{14}$ It has been commonly thought that overall survival has increased in the decade since that time, perhaps in response to improved patient care. However, 2 recent large trials ${ }^{2,5}$ both showed a 2-year survival of around $30 \%$ in the SOC arms. That value lies within the $95 \%$ confidence interval of the original Stupp trial. ${ }^{14}$ In contrast, both the 1- and 2-year survivals in the current trial fall outside the Stupp confidence intervals ${ }^{14}$ for those time points, suggesting statistical differences. That is, one can be $95 \%$ confident that survival in the present trial is statistically different from that which established the SOC. However, an SOC group treated at the same time as the TSC patients is needed to more extensively examine statistical differences, and that will be done in an upcoming Phase III trial.

It has been noted in a number of clinical trials that survival can be positively correlated with the extent of the initial resection, ${ }^{10}$ which means that those patients having inoperable tumors face an even bleaker prognosis. The biopsy-only patients undergo the same SOC as the other GBM patients. The current trial incorporating TSC into the SOC RT and TMZ for GBM enrolled essentially equal numbers of patients who had undergone complete resection (14) and no resection (15). These patients comprised approximately $50 \%$ of the 59 patients enrolled in the trial. The other $50 \%$ were patients who had undergone partial resection.

Experience would suggest that the patients who had complete resections would fare much better than those solely having needle biopsies. ${ }^{1}$ Survival at 2 years was quite similar for both groups in the present trial, however. Actually, both groups of patients showed better survival at 2 years than the overall survival rate seen with the historical controls. It should be noted that in a rat model of GBM, ${ }^{11}$ TSC-treated tumors completely disappeared.

\section{Tumor Sizes}

The mean tumor area, as judged on contrast MRI, increased soon after treatment began (Fig. 2), suggesting a reduced value for the median time for progression-free survival (PFS), another often-used end point in GBM trials. Indeed, PFS, as reported by the investigators, was short in the current trial with the median time to progression occurring at 3.3 months.

However, tumor progression over the duration of the

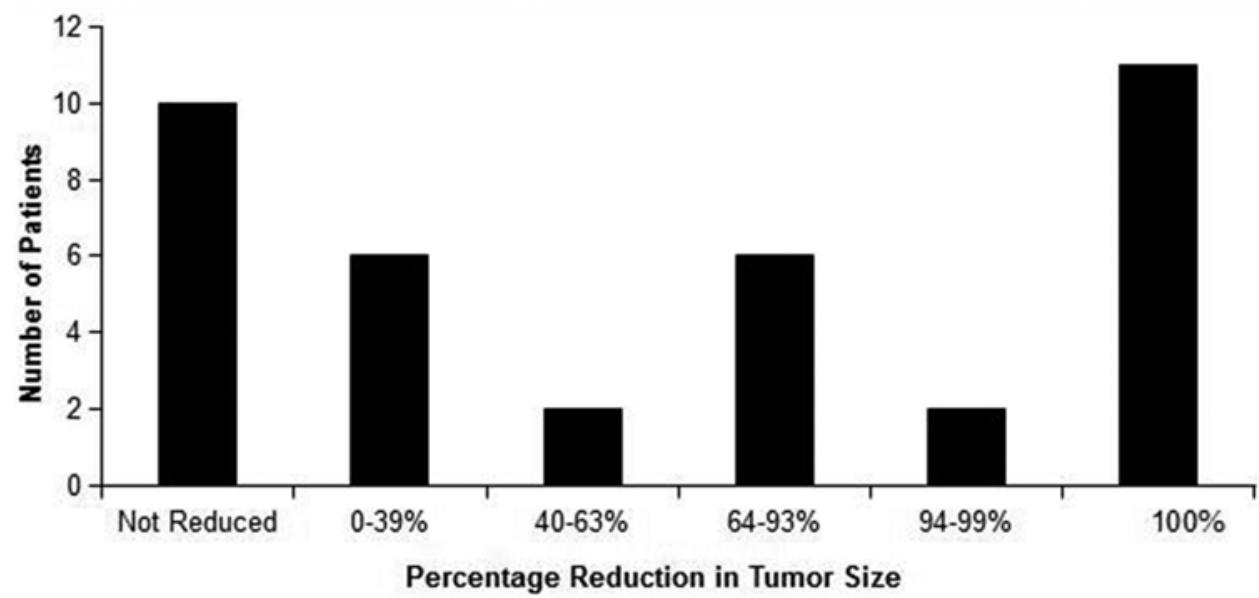

FIG. 3. Tumor size reductions with TSC as a radiosensitizer. 


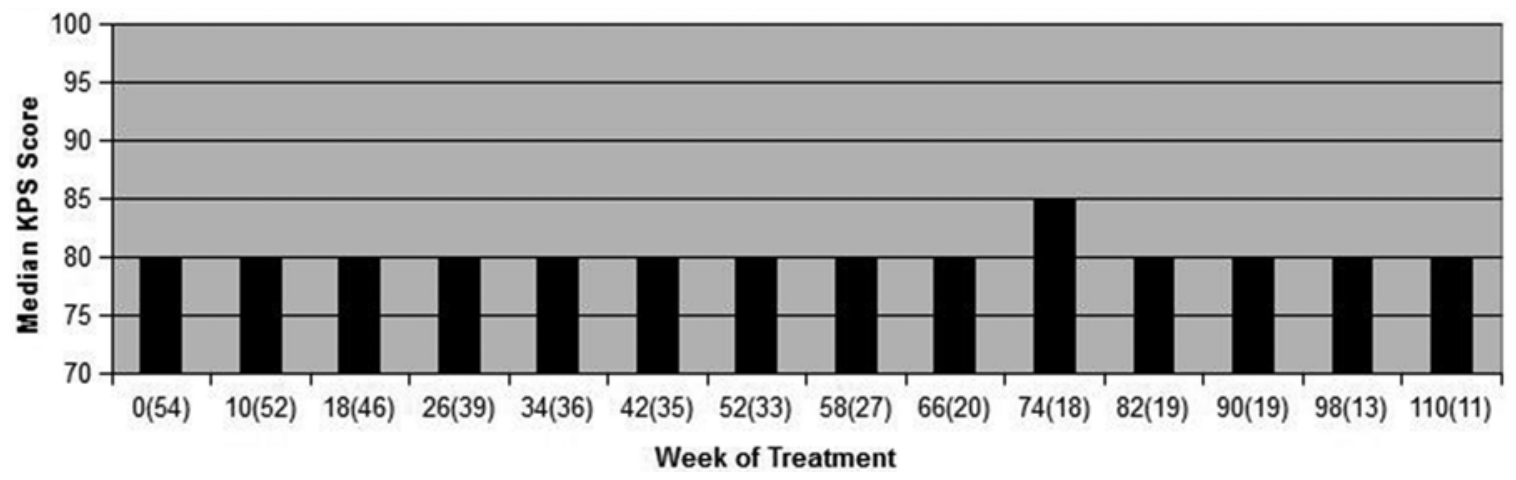

FIG. 4. Median KPS scores for the duration of the trial. Numbers in parentheses on the $x$-axis represent the number of patients whose data were reported by the clinical sites and do not necessarily correspond to the number of patients alive at the time; numbers outside the parentheses represent the number of weeks.

trial, as judged by contrast MRI, may require additional consideration. ${ }^{15}$ In looking at the MRI studies for the first 3 patient visits in our trial, we found that the mean MRI contrast area for tumor-bearing patients increased at 10 weeks but decreased at the third patient visit. This does not represent active tumor growth and shrinkage. Instead, this behavior is indicative of the phenomenon known as "pseudoprogression," 15 in which the increase seen on contrast MRI is not attributable to progression of an actively growing tumor. Rather, it is thought that the increase in size is due to the death of active tumor tissue and the swelling of subsequently formed necrotic tissue. Pseudoprogression is a relatively new phenomenon, having been seen only after introducing TMZ into the SOC, and is not completely understood. The data would suggest that there was considerable pseudoprogression in the current trial.

The reduction in tumor sizes (Fig. 3) is probably one of the most remarkable results of this study. There appear to be no published records of GBM tumors getting smaller with which to compare our results. This may seem surprising; however, anecdotal evidence from medical personnel connected to SOC RT in GBM suggests that the maximum tumor regression seen during RT is around
$20 \%-25 \%$. Thus, the disappearance of tumors during this trial in almost $20 \%$ of the full-dose patients would appear to emphasize the beneficial use of TSC for this indication.

\section{Patient Status During the Trial}

Two measures concerned the status of patients during this trial: KPS scores and QOL self-reported questionnaires. More data were reported for the KPS scores. The QOL questionnaires depend on compliance, and records of these data diminished during the latter stages of the trial.

\section{KPS Score}

The median KPS scores for the entire trial (Fig. 4) were always 80 or above. A score of 80 is defined as "normal activity with effort; some signs or symptoms of disease." Thus, the majority of patients were able to retain reasonably normal activity throughout the trial.

The KPS scores around and during the interval when TSC was administered (Weeks 1-6) are shown in Fig. 5. The median scores rose during those 6 weeks of TSC dosing but returned to baseline after the TSC dosing was

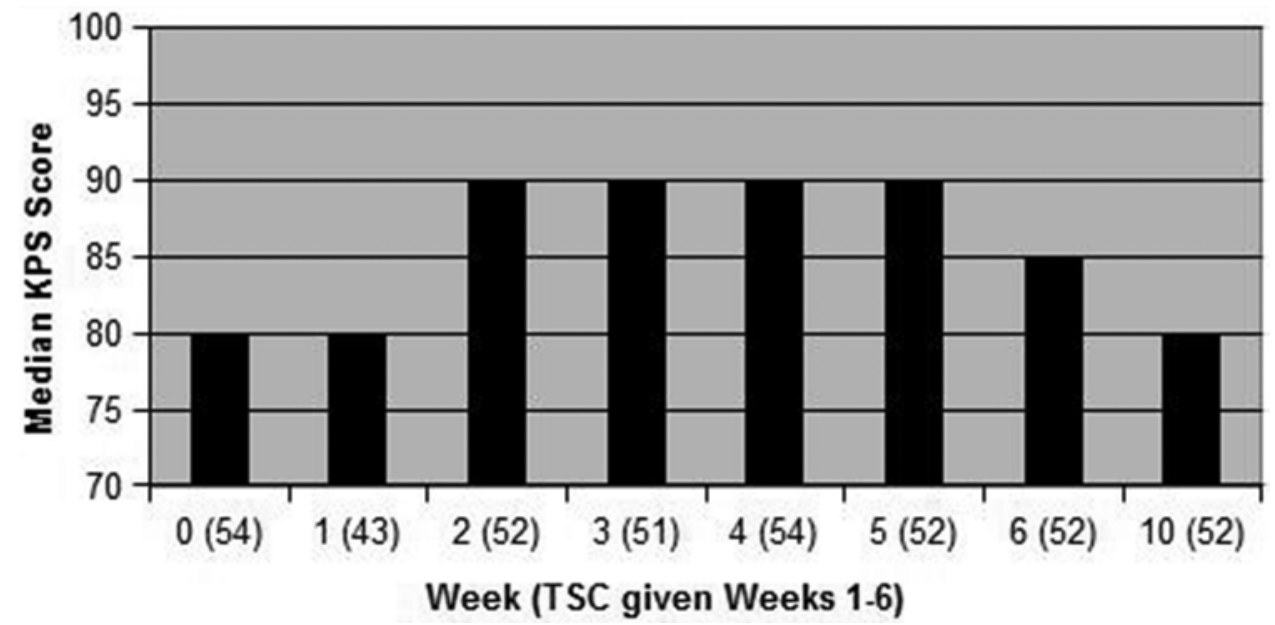

FIG. 5. Median KPS scores during TSC dosing. Numbers in parentheses on the $x$-axis represent the number of patients whose data were reported by the clinical sites and do not necessarily correspond to the number of patients alive at the time; numbers outside the parentheses represent the number of weeks. 


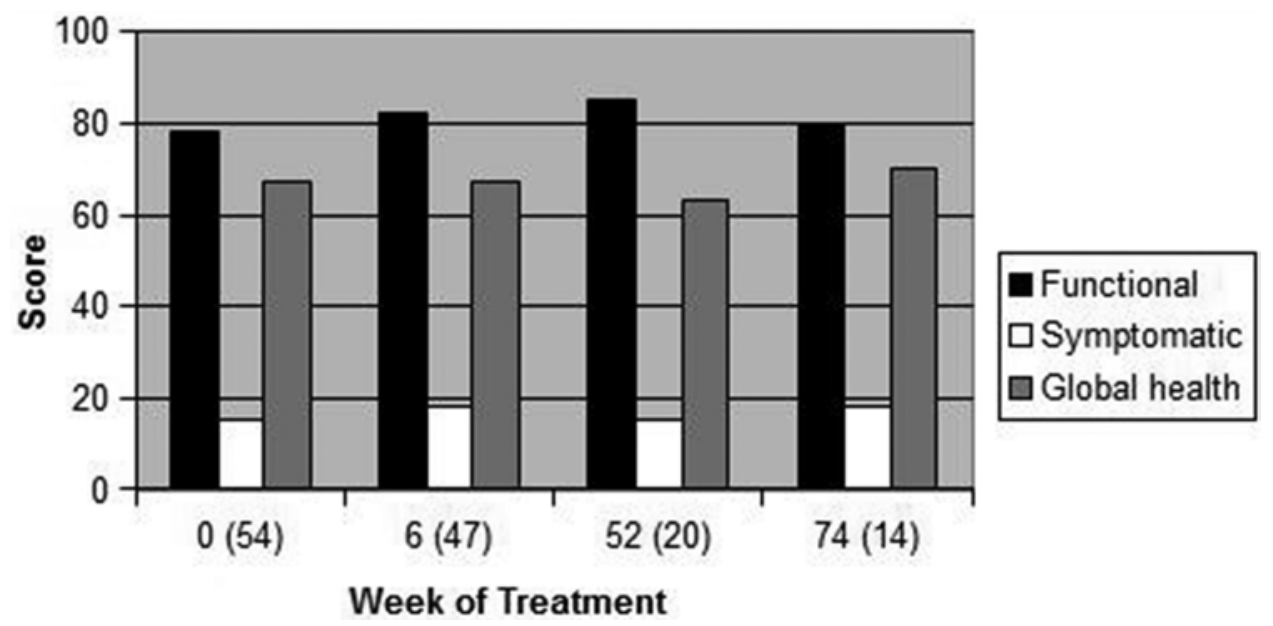

FIG. 6. Quality of life scores. Numbers in parentheses on the x-axis represent the number of patients whose data were reported by the clinical sites and do not necessarily correspond to the number of patients alive at the time; numbers outside the parentheses represent the number of weeks.

complete. This result would suggest that TSC is beneficial for patients when dosed with TMZ. As noted previously, TMZ dosing did not produce any evidence of myelosuppression, which may account for the higher KPS scores during this time.

\section{Quality of Life}

Quality of life was judged by the answers on 2 patient questionnaires. The results of one of those questionnaires, the EORTC QLQ-C30, for various periods of time during the trial (times when data compliance was better) are provided in Fig. 6. This questionnaire is helpful in that it allows one to look at 3 separate facets of patient life: functional, symptomatic, and global health. These separate scores were fairly constant throughout the trial, a trend also seen in the KPS scores. Both functional and global health aspects showed high scores, indicating that both were good, and symptomatic scores were low, indicating that relatively few symptoms were present.

\section{More About TSC}

Trans sodium crocetinate is a completely novel drug, and its mechanism of action is based on previous studies designed to identify the most significant resistance to oxygen movement in its pathway from the red blood cells to the tissues. Major resistance was proposed to come from the blood plasma, ${ }^{6,16}$ which is primarily composed of water, and water molecules are attracted to one another through hydrogen bonds. Increasing the number of hydrogen bonds among the water molecules will cause the water to become more structured on a microscopic scale, resulting in a decrease in its density. Chemical compounds that, when added to water, cause an increase in the water hydrogen bonding are called "kosmotropes." Trans sodium crocetinate, through its hydrophobic intermolecular forces, causes more hydrogen bonds to form among water molecules ${ }^{13}$ and thus can be considered to be a kosmotropic compound.

The physical changes caused by TSC, in essence, "open up" the water phase, allowing oxygen to diffuse more quickly from the red cells to the vascular wall. Thus, over time, the oxygen concentration at the vascular wall increases. That, in turn, causes an increase in the oxygen gradient between the vascular wall and the tissues. This gradient is especially pronounced if the tissues at the other end of the gradient have lower oxygen concentrations; that is, they are hypoxic. It is this increased oxygen gradient that is the driving force for more oxygen to diffuse from the blood vessels to the tissues.

Thus, TSC is quite unlike the majority of pharmaceutical compounds. It acts purely through physical chemical changes in the blood plasma. It has no effect on genes other than those that would be caused by increased oxygen itself. ${ }^{4}$ It should prove to be useful for alleviating hypoxia in any tissues since it acts on a systemic basis.

The chemotherapeutic portion of the SOC for GBM begins 1 month after RT is finished. It involves 6 monthly cycles during which larger doses of TMZ are given for 1 week, followed by 3 weeks of rest. It has also been suggested that increased oxygen may promote better results during chemotherapy; thus, extending TSC dosing to this portion of the SOC may also prove to be beneficial. This extended dosing of TSC is being planned in an upcoming Phase III clinical trial.

\section{Conclusions}

The antihypoxia drug TSC appears to be beneficial when added to the SOC for GBM. This trial showed higher overall survival at 1 and 2 years than that in the trial establishing the SOC for GBM. Greater survival rates were also seen for patients having only needle biopsies initially, a subgroup thought to fare worse than patients having resections. Considerable pseudoprogression appeared to occur in patients, as judged by tumor size changes during the first 18 weeks of the trial. In addition, it was shown that a considerable number of tumors became smaller. Quality of life did not diminish during the trial, and no serious adverse events were attributed to TSC. In fact, TSC has not resulted in a serious adverse event to date in 3 clinical trials involving over 150 patients. 


\section{Appendix}

Participating Clinical Sites and Principal Investigators

Barrow Neurological Institute, Phoenix, Arizona, Dr. William R. Shapiro; Baylor University Medical Center, Dallas, Texas, Dr. Karen Fink; Cedars-Sinai Medical Center, Los Angeles, California, Dr. Surasak Phuphanich; Emory University Medical Center, Atlanta, Georgia, Dr. H.-K. G. Shu; Forsyth Medical Center, Winston-Salem, North Carolina, Dr. Volker and Dr. W. W. Stieber; George Washington University Medical Center, Washington, DC, Dr. Jonathan H. Sherman; Medical College of Wisconsin, Milwaukee, Wisconsin, Dr. Mark G. Malkin and Dr. Jennifer M. Connelly; Medical University of South Carolina, Charleston, South Carolina, Dr. Pierre Giglio and Dr. Scott M. Lindhorst; Memorial HermannTexas Medical Center, Houston, Texas, Dr. Jay-Jiguang Zhu; North Shore University Health System, Manhasset, New York, Dr. Michael Schulder; Northwestern University Medical School, Chicago, Illinois, Dr. Jeffrey J. Raizer; Pennsylvania State Medical Center, Hershey, Pennsylvania, Dr. Jonas M. Sheehan; Rush University Medical Center, Chicago, Illinois, Dr. Robert Aiken; University of Arkansas Medical Center, Little Rock, Arkansas, Dr. Christopher P. Cifarelli and Dr. Shirley Ong; University of Florida, Gainesville, Florida, Dr. Erin Dunbar and Dr. Gregory J. A. Murad; University of Kentucky Medical Center, Lexington, Kentucky, Dr. John L. Villano; University of Southern California, Norris Cancer Center, Los Angeles, California, Dr. Naveed Wagle; and University of Virginia Medical Center, Charlottesville, Virginia, Dr. James M. Larner and Dr. Jason P. Sheehan.

\section{References}

1. Almeida JP, Chaichana KL, Rincon-Torroella J, QuinonesHinojosa A: The value of extent of resection of glioblastomas: clinical evidence and current approach. Curr Neurol Neurosci Rep 15:517, 2015

2. Chinot OL, Wick W, Mason W, Henriksson R, Saran F, Nishikawa R, et al: Bevacizumab plus radiotherapy-temozolomide for newly diagnosed glioblastoma. N Engl J Med 370:709-722, 2014

3. Gainer JL: Trans-sodium crocetinate for treating hypoxia/ischemia. Expert Opin Investig Drugs 17:917-924, 2008

4. Gainer JL, Lankford MF: Increasing oxygen in hypoxic tumors. Clin Exp Pharmacol 1:103, 2011

5. Gilbert MR, Dignam JJ, Armstrong TS, Wefel JS, Blumenthal DT, Vogelbaum MA, et al: A randomized trial of bevacizumab for newly diagnosed glioblastoma. N Engl J Med 370:699-708, 2014

6. Holland RAB, Shibata H, Scheid P, Piiper J: Kinetics of O2 uptake and release by red cells in stopped-flow apparatus: effects of unstirred layer. Respir Physiol 59:71-91, 1985

7. London South Bank University: Water Structure and Science. (http://www1.lsbu.ac.uk/water/kosmotropes_ chaotropes.html) [Accessed March 18, 2016]

8. Mohler ER III, Gainer JL, Whitten K, Eraso LH, Thanaporn PK, Bauer T: Evaluation of trans sodium crocetinate on safety and exercise performance in patients with peripheral artery disease and intermittent claudication. Vasc Med 16:346-353, 2011

9. Roy JW, Graham MC, Griffin AM, Gainer JL: A novel fluid resuscitation therapy for hemorrhagic shock. Shock 10:213217, 1998

10. Saito K, Mukasa A, Narita Y, Tabei Y, Shinoura N, Shibui S, et al: Toxicity and outcome of radiotherapy with concomitant and adjuvant temozolomide in elderly patients with glioblastoma: a retrospective study. Neurol Med Chir (Tokyo) 54:272-279, 2014

11. Sheehan J, Cifarelli CP, Dassoulas K, Olson C, Rainey J, Han $\mathrm{S}$ : Trans-sodium crocetinate enhancing survival and glioma response on magnetic resonance imaging to radiation and temozolomide. J Neurosurg 113:234-239, 2010

12. Sheehan JP, Shaffrey ME, Gupta B, Larner J, Rich JN, Park DM: Improving the radiosensitivity of radioresistant and hypoxic glioblastoma. Future Oncol 6:1591-1601, 2010

13. Stennett AK, Dempsey GL, Gainer JL: Trans-sodium crocetinate and diffusion enhancement. J Phys Chem B 110:18078-18080, 2006

14. Stupp R, Mason WP, van den Bent MJ, Weller M, Fisher $\mathrm{B}$, Taphoorn MJ, et al: Radiotherapy plus concomitant and adjuvant temozolomide for glioblastoma. $\mathbf{N}$ Engl J Med 352:987-996, 2005

15. Van Mieghem E, Wozniak A, Geussens Y, Menten J, De Vleeschouwer S, Van Calenbergh F, et al: Defining pseudoprogression in glioblastoma multiforme. Eur J Neurol 20:1335-1341, 2013

16. Yamaguchi K, Nguyen-Phu D, Scheid P, Piiper J: Kinetics of $\mathrm{O} 2$ uptake and release by human erythrocytes studied by a stopped-flow technique. J Appl Physiol (1985) 58:12151224,1985

\section{Disclosures}

This clinical trial was financed by Diffusion Pharmaceuticals LLC, Charlottesville, VA, and was independently conducted by INC Research, Raleigh, NC. Drs. Gainer and Jones are officers of Diffusion Pharmaceuticals, but all data, results, and graphs were done by INC as work for hire, and dissemination of these are done through the scientific offices of Diffusion. None of the authors will financially gain from this study since TSC is an experimental drug and is not commercially available.

\section{Author Contributions}

Conception and design: all authors. Acquisition of data: Sheehan, Larner. Drafting the article: Gainer. Critically revising the article: Gainer, Sheehan. Approved the final version of the manuscript on behalf of all authors: Gainer.

\section{Correspondence}

John L. Gainer, Diffusion Pharmaceuticals LLC, 2020 Avon

Court,\#4, Charlottesville, VA 22902.email: jgainer@ diffusionpharma.com. 